Tôhoku Math. Journ.

27 (1975), 69-74.

\title{
EXISTENCE OF ALMOST PERIODIC SOLUTIONS BY LIAPUNOV FUNCTIONS
}

\author{
F. NAKAJIMA
}

(Received December 8, 1973)

1. Introduction. The existence of almost periodic solutions of almost periodic systems has been studied by many authors. Generally, the existence of a bounded solution does not imply the existence of almost periodic solutions [4]. To obtain almost periodic solutions, we need additional conditions, for example, separation conditions and stability conditions. Another approach is to assume the existence of a Liapunov function with some properties ([2], [5]). Relationships between separation conditions and stability conditions have been discussed by the author [3].

In this paper, by assuming the existence of some Liapunov function, we shall obtain an existence theorem for an almost periodic solution, which improves Fink and Seifert's result [2] and proves Yoshizawa's result [5] as a corollary.

We denote by $R^{n}$ the Euclidean $n$-space and set $R=R^{1}$ and $R^{+}=$ $[0, \infty)$. Let $|x|$ be the Euclidean norm of $x \in R^{n}$.

2. Theorem and some remarks. Consider the almost periodic system

$$
x^{\prime}=f(t, x) \quad\left({ }^{\prime}=d / d t\right),
$$

where $x, f \in R^{n}$ and $f(t, x)$ is defined on $R \times D, D$ open set of $R^{n}$, and is almost periodic in $t$ uniformly for $x \in D$. The following theorem is an improvement of Fink and Seifert's result [2].

THEOREM. Suppose that the system (2.1) has a solution $\phi(t)$ such that $\phi(t) \in K$ on $R^{+}$, where $K$ is a compact subset of $D$, and assume that there exists a continuous scalar function $V(t, x)$ defined on $R^{+} \times D$, which satisfies the following conditions:

(i ) $V(t, \phi(t))$ is bounded on $R^{+}$,

(ii) $|V(t, x)-V(t, y)| \leqq L|x-y|$ for $x, y \in S, t \in R^{+}$, where $S$ is any compact subset of $D$ and $L$ may depend on $S$,

(iii) $\dot{V}(t, x) \geqq a(|x-\phi(t)|)$, where $a(r)$ is continuous and positive definite and

$$
\dot{V}(t, x)=\varlimsup_{h \rightarrow+0} \frac{1}{h}\{V(t+h, x+h f(t, x))-V(t, x)\} .
$$


Then the system (2.1) has a unique almost periodic solution in $D$ whose module is contained in the module of $f(t, x)$.

The proof shall be given in the next section. In order to obtain a unique almost periodic solution in $K$, Fink and Seifert have assumed the following conditions; in addition to the conditions in our theorem, $V(t, x)$ is defined on $R \times D$ and is continuous in $t$ uniformly for $(t, x) \in R \times S$ for each compact subset $S$ of $D$, and $V(t, \phi(t))=0$. Our theorem shows that we can drop these conditions and furthermore we can verify the uniqueness in $D$ of the almost periodic solution. As will be seen from the example below, the uniqueness of the almost periodic solutions in any compact subset of $D$ does not necessarily imply the uniqueness in $D$.

Consider

$$
\left\{\begin{array}{l}
x^{\prime}=\left(1-\frac{x-\phi(t)}{3-\phi(t)}\right) \phi^{\prime}(t) \\
y^{\prime}=(x-\phi(t))^{2}(x-3)^{2}+y^{2},
\end{array}\right.
$$

where $\phi(t)=\sin t+\sin \sqrt{2} t$. Let $D=(-2, \infty) \times(-\infty, \infty)$. Then there are exactly two almost periodic solutions in $D$, that is, $\{x=\phi(t), y=0\}$ and $\{x=3, y=0\}$. However, any compact subset of $D$ contains at most one almost periodic solution $\{x=3, y=0\}$, because $\inf _{t \in R} \phi(t)=-2$.

In our theorem, we have to know what $\phi(t)$ is. However, there is often a case where we know only the existence of a compact solution of (2.1). For such a case, the following corollary is useful and it also improves Yoshizawa's result [5], except the result on stability.

COROLLARY. Suppose that there exists a continuous scalar function $V(t, x, y)$ defined on $R^{+} \times D \times D$ which satisfies the following conditions:

(i ) $V(t, x, x)$ is bounded for $t \in R^{+}, x \in S$, where $S$ is any compact subset of $D$,

(ii) $\left|V\left(t, x_{1}, y_{1}\right)-V\left(t, x_{2}, y_{2}\right)\right| \leqq L\left\{\left|x_{1}-x_{2}\right|+\left|y_{1}-y_{2}\right|\right\}$ for $t \in R^{+}, x_{1}$, $x_{2}, y_{1}, y_{2} \in S$, where $L$ may depend on $S$,

(iii) $\dot{V}(t, x, y) \geqq a(|x-y|)$, where $a(r)$ is continuous and positive definite and

$$
\dot{V}(t, x, y)=\varlimsup_{h \rightarrow+0} \frac{1}{h}\{V(t+h, x+h f(t, x), y+h f(t, y))-V(t, x, y)\} .
$$

Moreover, assume that the system (2.1) has a solution which remains in a compact subset of $D$ for $t \geqq 0$.

Then the system (2.1) has a unique almost periodic solution in $D$ whose module is contained in the module of $f(t, x)$. 
Let $\phi(t)$ be a given compact solution and consider $V(t, x, \phi(t))$ as the Liapunov function in Theorem. Then this corollary follows immediately from our theorem.

3. Proof of Theorem. The following lemma is well known (cf. [1]).

Lemma. Let $S$ be a compact subset of $D$. For each $g$ in the hull of $f$, assume that the system

$$
x^{\prime}=g(t, x)
$$

has one and only one solution which remains in $S$ for all $t \in R$.

Then the system (2.1) has an almost periodic solution whose module is contained in the module of $f(t, x)$.

Under our assumption, we shall show that for each $g$ in the hull of $f$, the system (3.1) has one and only one solution in $K$ for all $t \in R$. Since $f(t, x)$ is almost periodic in $t$, there is a sequence $\left\{t_{k}\right\}$ such that $t_{k} \rightarrow \infty$ as $k \rightarrow \infty$ and

$$
f\left(t+t_{k}, x\right) \rightarrow g(t, x)
$$

uniformly on $R \times K$ as $k \rightarrow \infty$. Since $\left\{\phi\left(t+t_{k}\right)\right\}_{k=1}^{\infty}$ is uniformly bounded and equicontinuous on any compact interval in $R$, we can assume that

$$
\phi\left(t+t_{k}\right) \rightarrow \psi(t)
$$

uniformly on any compact interval in $R$ as $k \rightarrow \infty$.

Then $\psi(t) \in K$ for all $t \in R$ and $\psi(t)$ is a solution of (3.1). We shall show that if system (3.1) has a solution $x(t)$ such that $x(t) \in K$ for all $t \in R$, then $x(t)=\psi(t)$ for all $t \in R$.

Let $V_{k}$ be defined by

$$
V_{k}(t)=V\left(t+t_{k}, x(t)\right) \text { for } t \geqq-t_{k},
$$

and set

$$
D^{+} V_{k}(t)=\varlimsup_{h \rightarrow+0} \frac{1}{h}\left\{V\left(t+t_{k}+h, x(t+h)\right)-V(t, x(t))\right\} .
$$

Then, by condition (ii), we have

$$
D^{+} V_{k}(t) \geqq \dot{V}\left(t+t_{k}, x(t)\right)-A_{k}(t),
$$

where $A_{k}(t)=L\left|g(t, x(t))-f\left(t+t_{k}, x(t)\right)\right|$ and $L=L\left(K^{\prime}\right)$ is the constant in condition (ii) for $K^{\prime}, K^{\prime}$ compact neighbourhood of $K$. Clearly we have

$$
\lim _{k \rightarrow \infty} A_{k}-(t)=0 \quad \text { uniformly on } R .
$$


By condition (iii), we have

$$
D^{+} V_{k}(t) \geqq a\left(\left|x(t)-\phi\left(t+t_{k}\right)\right|\right)-A_{k}(t) .
$$

On any interval $[b, c]$, if $k$ is sufficiently large so that $b+t_{k} \geqq 0$, we obtain

$$
V_{k}(c)-V_{k}(b) \geqq \int_{b}^{c} a\left(\left|x(s)-\phi\left(s+t_{k}\right)\right|\right) d s-\int_{b}^{c} A_{k}(s) d s .
$$

By conditions (i) and (ii), there exists a $B>0$ such that

$$
\left|V_{k}(c)-V_{k}(b)\right|=\left|V\left(c+t_{k}, x(c)\right)-V\left(b+t_{k}, x(b)\right)\right| \leqq B \text { for all } k \text {. }
$$

Therefore we have

$$
\int_{b}^{c} a\left(\left|x(s)-\phi\left(s+t_{k}\right)\right|\right) d s-\int_{b}^{c} A_{k}(s) d s \leqq B .
$$

Letting $k \rightarrow \infty$, it follows from (3.3) and (3.4) that

$$
\int_{b}^{c} a(|x(s)-\psi(s)|) d s \leqq B \text {. }
$$

Since $b$ and $c$ are arbitrary, we have

$$
\int_{-\infty}^{\infty} a(|x(s)-\psi(s)|) d s \leqq B,
$$

and hence, there. exist sequences $\left\{\tau_{m}\right\}$ and $\left\{\sigma_{m}\right\}$ such that $\tau_{m} \rightarrow-\infty$, $\sigma_{m} \rightarrow+\infty$, as $m \rightarrow \infty$ and that $a\left(\left|x\left(\tau_{m}\right)-\psi\left(\tau_{m}\right)\right|\right) \rightarrow 0, a\left(\left|x\left(\sigma_{m}\right)-\psi\left(\sigma_{m}\right)\right|\right) \rightarrow 0$ as $m \rightarrow \infty$. This shows

$$
\left|x\left(\tau_{m}\right)-\psi\left(\tau_{m}\right)\right| \rightarrow 0, \quad\left|x\left(\sigma_{m}\right)-\psi\left(\sigma_{m}\right)\right| \rightarrow 0 \text { as } m \rightarrow \infty
$$

since $a(r)$ is continuous, positive definite and $\left|x\left(\tau_{m}\right)-\psi\left(\tau_{m}\right)\right|, \mid x\left(\sigma_{m}\right)-$ $\psi\left(\sigma_{m}\right) \mid$ are bounded.

In (3.5), let $b=\tau_{m}$ and $c=\sigma_{m}$. Then, if $k$ is sufficiently large so that $\tau_{m}+t_{k} \geqq 0$, we have

and

$$
V_{k}\left(\sigma_{m}\right)-V_{k}\left(\tau_{m}\right) \geqq \int_{\tau_{m}}^{\sigma_{m}} a\left(\left|x(s)-\phi\left(s+t_{k}\right)\right|\right) d s-\int_{\tau_{m}}^{\sigma_{m}} A_{k}(s) d s
$$

$$
\begin{aligned}
& \int_{\tau_{m}}^{\sigma_{m}} a\left(\left|x(s)-\phi\left(s+t_{k}\right)\right|\right) d s-\int_{\tau_{m}}^{\sigma_{m}} A_{k}(s) d s-V\left(\sigma_{m}+t_{k}, \phi\left(\sigma_{m}+t_{k}\right)\right) \\
& +V\left(\tau_{m}+t_{k}, \phi\left(\tau_{m}+t_{k}\right)\right) \\
\leqq & V_{k}\left(\sigma_{m}\right)-V_{k}\left(\tau_{m}\right)-V\left(\sigma_{m}+t_{k}, \phi\left(\sigma_{m}+t_{k}\right)\right)+V\left(\tau_{m}+t_{k}, \phi\left(\tau_{m}+t_{k}\right)\right) \\
\leqq & L\left\{\left|x\left(\sigma_{m}\right)-\phi\left(\sigma_{m}+t_{k}\right)\right|+\left|x\left(\tau_{m}\right)-\phi\left(\tau_{m}+t_{k}\right)\right|\right\} \\
\leqq & L\left\{\left|x\left(\sigma_{m}\right)-\psi\left(\sigma_{m}\right)\right|+\left|\psi\left(\sigma_{m}\right)-\phi\left(\sigma_{m}+t_{k}\right)\right|+\left|x\left(\tau_{m}\right)-\psi\left(\tau_{m}\right)\right|\right. \\
& \left.\quad+\left|\psi\left(\tau_{m}\right)-\phi\left(\tau_{m}+t_{k}\right)\right|\right\} .
\end{aligned}
$$


Hence, letting $k \rightarrow \infty$, we can see that for a fixed $m$,

$$
\begin{aligned}
& \quad \int_{\tau_{m}}^{\sigma_{m}} a(|x(s)-\psi(s)|) d s-\varlimsup_{k \rightarrow \infty}\left\{V\left(\sigma_{m}+t_{k}, \phi\left(\sigma_{m}+t_{k}\right)\right)\right. \\
& \left.\quad-V\left(\tau_{m}+t_{k}, \phi\left(\tau_{m}+t_{k}\right)\right)\right\} \\
& \leqq \\
& \quad L\left\{\left|x\left(\sigma_{m}\right)-\psi\left(\sigma_{m}\right)\right|+\left|x\left(\tau_{m}\right)-\psi\left(\tau_{m}\right)\right|\right\} .
\end{aligned}
$$

However, since $V(t, \phi(t))$ is bounded and $D^{+} V(t, \phi(t)) \geqq 0, V(t, \phi(t)) \rightarrow v_{0}$ as $t \rightarrow \infty$ for some constant $v_{0}$, and hence, (3.7) implies

$$
\int_{\tau_{m}}^{\sigma_{m}} a(|x(s)-\psi(s)|) d s \leqq L\left\{\left|x\left(\sigma_{m}\right)-\psi\left(\sigma_{m}\right)\right|+\left|x\left(\tau_{m}\right)-\psi\left(\tau_{m}\right)\right|\right\} .
$$

Letting $m \rightarrow \infty$, it follows from (3.6) that

$$
\int_{-\infty}^{\infty} a(|x(s)-\psi(s)|) d s=0,
$$

which implies $a(|x(s)-\psi(s)|)=0$, that is, $x(s)=\psi(s)$ for all $s \in R$.

Now we shall show the uniqueness of the almost periodic solution in $D$. Let $\left\{t_{k}\right\}$ be a sequence such that $t_{k} \rightarrow \infty, f\left(t+t_{k}, x\right) \rightarrow f(t, x)$ uniformly on $R \times S, S$ any compact set in $D$, and $\phi\left(t+t_{k}\right) \rightarrow \psi(t)$ uniformly on any compact interval in $R$ as $k \rightarrow \infty$. Then $\psi(t) \in K$ for all $t \in R$ and, as was seen above, $\psi(t)$ is the unique solution in $K$ of system (2.1). Thus $\psi(t)$ is an almost periodic solution of system (2.1). Therefore it is sufficient to show that $\psi(t)=p(t)$ for any almost periodic solution $p(t)$ of $(2.1)$ in $D$.

Suppose that there exists an almost periodic solution $p(t)$ of (2.1) such that $p(t) \in D$ for all $t \in R$ and $\left|p\left(t_{0}\right)-\psi\left(t_{0}\right)\right|=\varepsilon$ at some $t_{0} \in R$ for some $\varepsilon>0$. Since $p\left(t_{0}\right) \in D$, there exists an open set $O$ with the compact closure $\bar{O} \subset D$ such that $p\left(t_{0}\right) \in O \subset \bar{O} \subset D$. Since $p(t)$ is almost periodic, there exists a sequence $\left\{\sigma_{m}\right\}$ such that $\sigma_{m} \rightarrow \infty$ as $\mathrm{m} \rightarrow \infty$ and $p\left(\sigma_{m}\right) \in \bar{O}$ for all $m$.

Let $V_{k}(t)=V\left(t+t_{k}, p(t)\right)$. Then, by the same argument as used in obtaining (3.5), we have

$$
V_{k}\left(\sigma_{m}\right)-V_{k}\left(t_{0}\right) \geqq \int_{t_{0}}^{\sigma_{m}} a\left(\left|p(t)-\phi\left(t+t_{k}\right)\right|\right) d t-\int_{t_{0}}^{\sigma_{m}} A_{k}(m, t) d t,
$$

where $A_{k}(m, t)=L_{m}\left|f\left(t+t_{k}, p(t)\right)-f(t, p(t))\right|$ and $L_{m}$ may depend on a compact set $K_{m}$ in $D$ which is a neighbourhood of the compact set $\left\{p(t) ; t_{0} \leqq t \leqq \sigma_{m}\right\}$. Clearly, for a fixed $m$,

$$
\lim _{k \rightarrow \infty} A_{k}(m, t)=0 \text { uniformly for } t \in\left[t_{0}, \sigma_{m}\right] \text {. }
$$

Since $p\left(\sigma_{m}\right) \in \bar{O}$ and we have conditions (i), (ii), there exists a $B>0$ such that 


$$
\left|V_{k}\left(\sigma_{m}\right)-V_{k}\left(t_{0}\right)\right| \leqq B \text { for all } m \text {. }
$$

Letting $k \rightarrow \infty$ in (3.8), we have

which implies

$$
\int_{t_{0}}^{o_{m}} a(|p(t)-\psi(t)|) d t \leqq B
$$

$$
\int_{t_{0}}^{\infty} a(|p(t)-\psi(t)|) d t \leqq B
$$
that

Since $p(t)-\psi(t)$ is almost periodic, there exists a sequence $\left\{\tau_{m}\right\}$ such

$$
\left|p\left(t_{0}\right)-\psi\left(t_{0}\right)-p\left(\tau_{m}\right)+\psi\left(\tau_{m}\right)\right|<\varepsilon / 3 \text { for all } m
$$

$$
\tau_{m} \rightarrow \infty \text { as } m \rightarrow \infty, \quad \tau_{m}+2<\tau_{m+1} .
$$

The uniform continuity of $p(t)-\psi(t)$ implies the existence of a $\delta, 0<\delta<1$, such that

$$
\left|p(t)-\psi(t)-p\left(\tau_{m}\right)+\psi\left(\tau_{m}\right)\right|<\varepsilon / 3 \text { for } \tau_{m}-\delta<t<\tau_{m}+\delta
$$

From (3.10), (3.12) and $\left|p\left(t_{0}\right)-\psi\left(t_{0}\right)\right|=\varepsilon$, it follows that

$$
\varepsilon / 3<|p(t)-\psi(t)|<5 \varepsilon / 3 \text { for } \tau_{m}-\delta<t<\tau_{m}+\delta \text { and all } m .
$$

Let

Then we have

$$
a_{0}=\min \{a(r) ; \varepsilon / 3 \leqq r \leqq 5 \varepsilon / 3\}(>0) .
$$

$$
B \geqq \sum_{m=1}^{\infty} \int_{\tau_{m}-\delta}^{\tau_{m}+\delta} a(|p(t)-\psi(t)|) d t \geqq \sum_{m=1}^{\infty} 2 \delta a_{0}=\infty
$$

since the intervals $\left(\tau_{m}-\delta, \tau_{m}+\delta\right)$ are disjoint by (3.11). This is a contradiction. Thus $p(t)=\psi(t)$. This completes the proof.

\section{REFERENCES}

[1] L. Amerio, Soluzioni quasi-periodiche, o limitate, di sistemi differenziali non lineari quasi-periodici o limitate, Ann. Mat. Pura. Appl., 39 (1955), 97-119.

[2] A. M. FinK AND G. SEIFERT, Liapunov functions and almost periodic solutions for almost periodic systems, J. Diff. Eqs., 5 (1969), 307-313.

[3] F. NAKaJima, Separation conditions and stability properties in almost periodic systems, Tôhoku Math. J., 26 (1974), 305-314.

[4] Z. OPIAL, Sur une equation différentielle presque-périodique sans solution presquepériodique, Bull. Acad. Polon. Sci. Sér. Sci. Math. Astronom. Phys., 9 (1961), 673-676.

[5] T. Yoshizawa, Extreme stability and almost periodic functional differential equations, Arch. Rational. Mech. Anal., 17 (1964), 148-170.

Mathematical INSTitute,

TÔHOKU UNIVERSITY,

SENDAI, JAPAN. 